\title{
Hipertrofia do músculo masseter e artrose: relato de caso
}

\author{
Masseter muscle hypertrophy and arthrosis: case report \\ Hipertrofia y artrosis muscular maseter: reporte de caso
}

Deise Karine Cavalcante Leão ${ }^{1 *}$, Ricardo Luiz Ferreira da Silva Junior ${ }^{1}$, Fernanda Braga Peixoto ${ }^{1}$.

\begin{abstract}
RESUMO
Objetivo: Descrever um caso de Hipertrofia do músculo masseter unilateral, associado a artrose. Detalhamento do caso: Paciente do gênero feminino, 45 anos, melanoderma, procurou atendimento odontológico em uma cidade de Alagoas, encaminhada por um ortopedista. A mesma queixava-se de um peso no lado esquerdo do rosto e dores de cabeça frequentes, revelando já ter sido submetida a um procedimento cirúrgico em consequência, denominado artroscopia. A osteoartrose, pode ser diagnosticada através de exames complementares como radiografia panorâmica e ressonância magnética. Este relato ilustra um caso raro de hipertrofia do músculo masseter, sintomática, grande volume no ramo da mandíbula, redução dos espaços articulares e perda das esfericidades dos côndilos mandibulares e que pode estar ligado também a fatores psicológicos como stress e nervosismo. Considerações finais: A conduta adotada para esse caso foi a prescrição e a utilização de colágeno tipo II e fisioterapia no músculo masseter, obtendo-se sucesso na diminuição da intensidade da dor e na frequência.
\end{abstract}

Palavras-chave: Hipertrofia, Músculo masseter, Osteoartrose.

\begin{abstract}
Objective: To describe a case of Hypertrophy of the unilateral masseter muscle, associated with arthrosis. Case details: Female patient, 45 years old, melanoderma, sought dental care in a city in Alagoas, referred by an Orthopedist. She complained of a weight on the left side of her face and frequent headaches, revealing that she had already undergone a surgical procedure as a result, called arthroscopy. Osteoarthritis can be diagnosed through complementary exams such as panoramic radiography and magnetic resonance imaging. This report illustrates a rare case of symptomatic masseter muscle hypertrophy, large volume in the mandible branch, reduction of joint spaces and loss of sphericity of the mandibular condyles and which may also be linked to psychological factors such as stress and nervousness. Final considerations: The approach adopted for this case was the prescription and use of type II collagen and physiotherapy in the masseter muscle, with success in decreasing pain intensity and frequency.
\end{abstract}

Keywords: Hypertrophy, Masseter muscle, Osteoarthritis.

\section{RESUMEN}

Objetivo: Describir un caso de hipertrofia del músculo masetero unilateral asociado a artrosis. Detalle del caso: Paciente femenina de 45 años con melanodermia, buscó atención dental en una ciudad de Alagoas, remitida por un ortopedista. Se quejaba de un peso en el lado izquierdo de la cara y frecuentes dolores de cabeza, revelando que ya se había sometido a un procedimiento quirúrgico como resultado, llamado artroscopia. La osteoartritis se puede diagnosticar mediante exámenes complementarios como la radiografía panorámica y la resonancia magnética. Este informe ilustra un caso raro de hipertrofia del músculo masetero, sintomático, gran volumen en la rama mandibular, reducción de los espacios articulares y pérdida de esfericidades de los cóndilos mandibulares y que también puede estar relacionado con factores psicológicos como el estrés y el nerviosismo. Consideraciones finales: El abordaje adoptado para este caso fue la prescripción y uso de colágeno tipo Il y fisioterapia en el músculo maseero, con éxito en la disminución de la intensidad y frecuencia del dolor.

Palabras clave: Hipertrofia, Musculo masetero, Osteoartritis.

${ }^{1}$ Centro Universitário Cesmac, Maceió - AL. *E-mail: deisekarine71@gmail.com 


\section{INTRODUÇÃO}

A hipertrofia do músculo masseter (HMM) é caracterizada pelo aumento anormal deste músculo, podendo ser unilateral ou bilateral, é considerada uma condição benigna rara, geralmente assintomática. Pode ser congênita ou adquirida, pela hipertrofia funcional. Em relação a HMM adquirida sua etiologia permanece desconhecida, porém alguns fatores são considerados contribuintes, como bruxismo, má oclusão, mastigação unilateral, distúrbios da articulação temporomandibular, distúrbios emocionais como estresse e nervosismo (ANEHOSUR MDSV, et al., 2020).

Existem cinco tipos de classificação da hipertrofia massetérica, são elas: Tipo I: Protuberância mínima, sem evidência clínica; Tipo II: uma protuberância localizada, evidente clinicamente; Tipo III: Protuberância dupla, apresentando dois feixes longitudinais separados, podendo ser do mesmo tamanho ou tamanhos diferentes; Tipo IV: Protuberância tripla, como três feixes longitudinais; Tipo V: Uma única protuberância excessiva, não delimitada ou difusa (GRACIA OPN, et al., 2021).

O diagnóstico correto da hipertrofia do músculo masseter é de extrema importância, já que existe uma série de patologias dentro de seu diagnóstico diferencial, incluindo tumores musculares, distúrbios de glândulas salivares, tumores e doenças inflamatórias da glândula parótida, miopatia intrínseca do músculo masseter, pseudo-hipertrofia devido a inflamação ou infiltração de gordura, neoplasias vasculares, lipossarcoma, rabdomiossarcoma, linfonodo metastático, linfangioma, linfadenite, miosite ossificante, leucemia infiltrativa ou linfoma (VALENCIA AR, et al., 2019; NAPHADE VV, et al., 2018).

Dessa forma, deve ser feito uma avaliação minuciosa, para chegar no correto diagnóstico, que deve ser obtido através da associação das informações adquiridas pela anamnese, exame clínico e exames complementares, como radiografias panorâmicas convencionais, tomografia computadorizada e ressonância magnética (SIMÃO RS, et al., 2014).

Em relação às características clínicas desse tipo de hipertrofia, existem alguns fatos que devem ser analisados, e que ajudaram a diferenciar essa patologia das demais citadas, se essa alteração de volume é condizente com uma massa uniforme de longa duração, que está localizada na região característica do músculo masseter, e que quando o paciente aperta os dentes ocorre uma contração muscular no local em que está essa proeminência, são sinais que fazem com que essa hipótese diagnóstica de hipertrofia do músculo masseter se torne ainda mais forte, porém o diagnóstico o final não deve ser fechado antes da avaliação de um exame imagiológico (NAPHADE VV, et al., 2018).

De acordo com a literatura não é necessário tratamento para HMM. Entretanto, existem algumas modalidades de tratamento, podendo ser cirúrgica ou não cirúrgica. A primeira inclui o uso de relaxante muscular, cuidados psiquiátricos e injeção da toxina botulínica do tipo A. A BoNT-A no músculo hipertrofiado, funciona pelo princípio de inibir a contração muscular, a inibição desse fenômeno ocorre, pois, tal toxina dificulta a liberação de acetilcolina, levando então a uma paralisia do músculo, como consequência ocorre à "atrofia por desuso" e logo a redução do volume muscular. Uma desvantagem desse método, é que o mesmo precisa ser repetido várias vezes, já que sua ação é temporária, voltando a sua condição inicial cerca de 6 meses depois da aplicação (AYHAN A, et al., 2018).

O método cirúrgico é caracterizado pela excisão de parte do músculo masseter, osteotomia da região do ângulo da mandíbula e remodelação da curvatura do osso, sob efeito de anestesia geral. De acordo com Wood, a abordagem intraoral deveria se deter somente a remoção do aumento ósseo do ângulo mandibular, sem a excisão de parte do músculo masseter. Em contrapartida, Tabrizi et al. defendeu uma abordagem que incluía tanto a osteotomia parcialmente monocortical e bicortical do ângulo mandibular, como a redução do músculo hipertrofiado (AYHAN A, et al., 2018). Um dos métodos que também está sendo utilizado atualmente, quando o paciente relata sintomatologia dolorosa, é a fisioterapia do masseter através da massagem desse músculo. A massagem geralmente obtém resultados positivos em relação a redução da dor, aumento da mobilidade articular, eliminação de aderências entre as fibras musculares, e a mesma produz um aumento da circulação local, gerando um relaxamento dessa região (HAGE YE, et al., 2013). 
A artrose é denominada como uma doença articular crônica, de caráter lento, progressivo e debilitante que representa um processo de degeneração gradual da cartilagem em uma articulação. Na articulação temporomandibular é perceptível a destruição do côndilo mandibular e da fossa mandibular. Geralmente seu desenvolvimento é associado a sobrecarga, bruxismo, mastigação unilateral, podendo também estar ligada a fatores genéticos e distúrbios internos. A mesma apresenta sintomatologia variável, podendo apresentar dor na ATM, crepitação, restrição da abertura bucal e até mesmo perda da função articular. Sua prevalência é maior no sexo feminino e ocorre com mais frequência ao decorrer da idade. Quando existe sintomatologia dolorosa, o tratamento inclui abordagens conservadoras, como uso de analgésicos, anti-inflamatórios orais, fisioterapia, exercícios isométricos, injeções intra-articulares, correção de anormalidade dentais e em alguns casos a suplementação de colágeno tipo II (SOARES IS, et al., 2019).

A osteoartrose é precedida pela osteoartrite, a osteoartrite é considerada um processo inflamatório de baixo nível, que acarreta a erosão e degradação da cartilagem articular e do osso subjacente. Enquanto as alterações ósseas estão ativas é denominada osteoartrite, mas a partir do momento em que ocorre a remodelação óssea, e essa condição se torna estável, mesmo que a morfologia óssea continue alterada, essa patologia passa a se chamar osteoartrose (SÁNCHEZ MT e BECERRA WB, 2020).

O objetivo desse estudo é relatar e discutir o caso de uma paciente que apresentava hipertrofia do músculo masseter associada a artrose da articulação temporomandibular.

\section{DETALHAMENTO DO CASO}

O termo de consentimento Livre e Esclarecido (TCLE) foi explicado a paciente, a mesma aceitou as condições e assinou o termo. Paciente do sexo feminino, V. L.F. S., 45 anos de idade, melanoderma, procurou atendimento odontológico em uma cidade de Alagoas encaminhada por um ortopedista. Em sua queixa, relatou sentir um peso muito grande no lado esquerdo do rosto, e frequentes dores de cabeça. Informou também ter sido submetida ao procedimento cirúrgico de artroscopia, em consequência.

Ao ser realizada a anamnese foi constatado que a paciente apresentava um bom estado geral de saúde. Durante o exame clínico extraoral foi observado uma grande assimetria facial, por conta de um aumento de volume na região massetérica e proeminência da região do ângulo mandibular esquerdo (Figura 1). Também se notou uma grande limitação do movimento de abertura de boca, no qual a abertura máxima era equivalente apenas a 12 milímetros (Figura 2).

Figura 1 - Assimetria facial. Notável discrepância do lado esquerdo em relação ao lado direito da face da paciente, devido a hipertrofia do músculo masseter esquerdo.

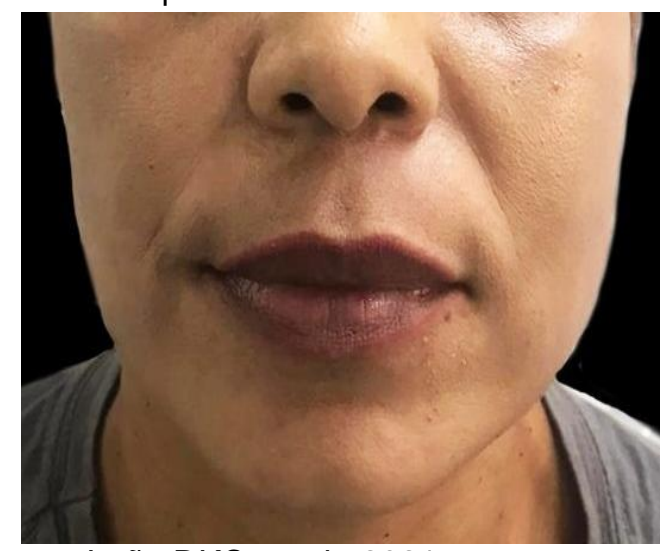

Fonte: Leão DKC, et al., 2021.
Figura 2 - Restrição da abertura bucal. Abertura máxima equivalente a 12 milímetros.

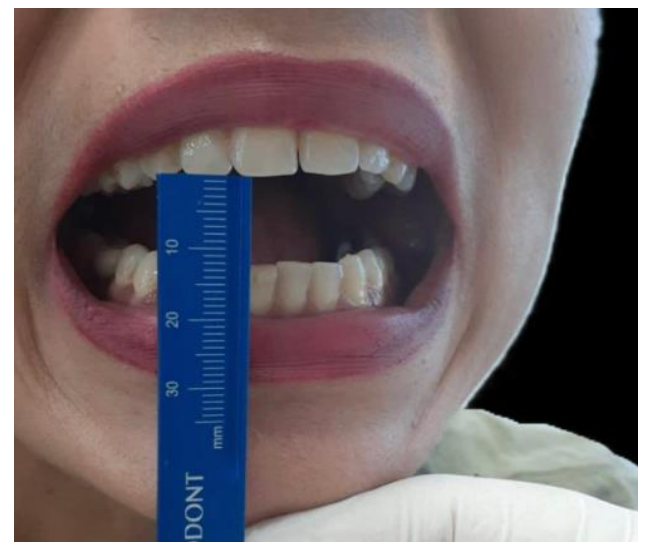

Fonte: Leão DKC, et al., 2021.

Na radiografia panorâmica foi confirmado a hipertrofia óssea no ramo da mandíbula do lado esquerdo decorrente da hiperestimulação do músculo masseter (Figura 3). E a avaliação da ressonância magnética revelou uma importante redução dos espaços das articulações temporomandibulares, juntamente com a 
perda das esfericidades dos côndilos mandibulares associado a osteoartrose (artrose), ainda na RM foi notado um afilamento da cartilagem articular e focos de edema subcondral (Figura 4).

Figura 3 - Radiografia panorâmica evidenciando radiopacidade compatível com hipertrofia óssea e de tecido mole (músculo masseter).

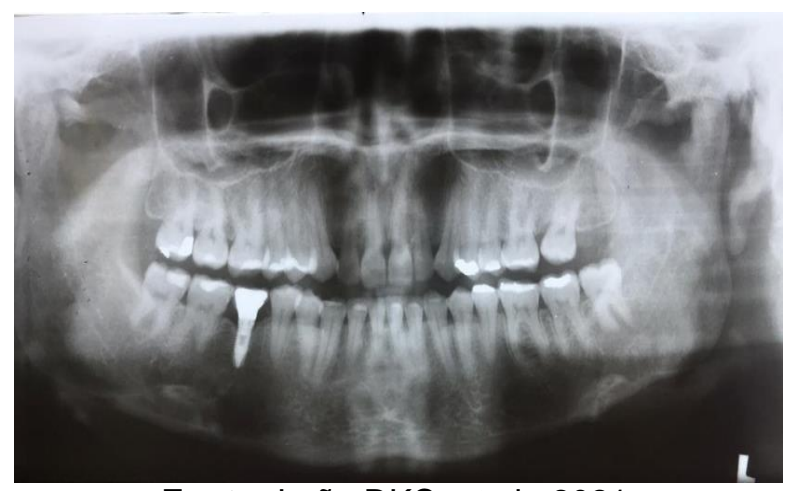

Fonte: Leão DKC, et al., 2021.

Figura 4 - Atrose (Osteofitose) condilar.

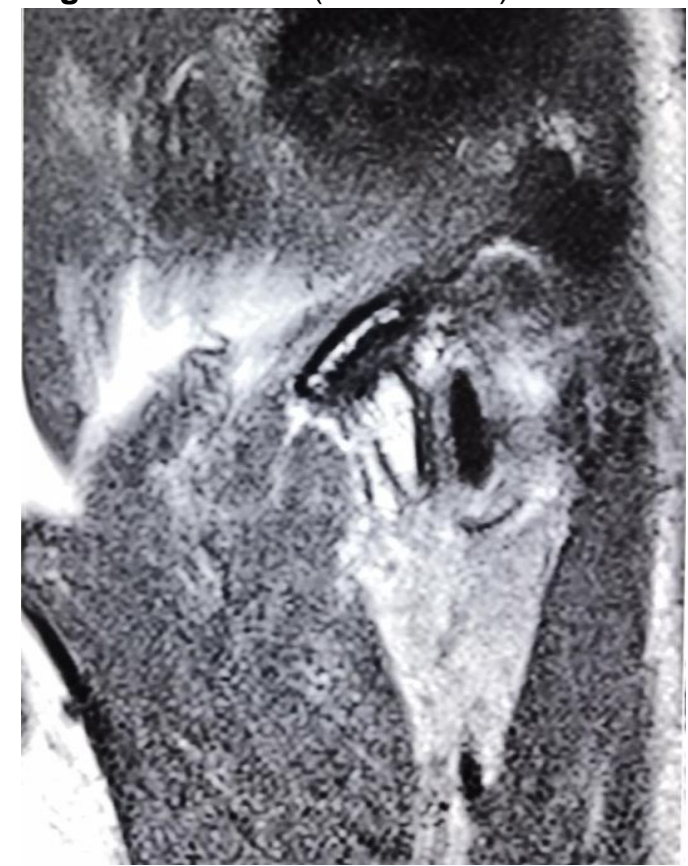

Fonte: Leão DKC, et al., 2021.

Por ser uma condição benigna, apesar de sua assimetria, foi discutido com a paciente, e a mesma não achou necessário interferir no quesito estético, almejando apenas a interrupção dos sintomas. Sendo assim, a conduta adotada foi prescrever o uso de colágeno tipo II, uma vez ao dia, durante 30 dias, associado à fisioterapia no músculo masseter, realizando um ciclo de dez repetições, três vezes ao dia. Após uma semana da primeira consulta, a paciente retornou à clínica e foram constatadas melhoras significativas da intensidade e frequência da dor.

\section{DISCUSSÃO}

Nesse relato, a hipertrofia óssea do músculo masseter, foi encontrada em uma paciente do sexo feminino, 45 anos de idade, melanoderma, em relação ao gênero o dado não difere da literatura, na qual diz que não há predileção por sexo. Entretanto, em relação à época de vida, a literatura afirma que a doença acomete mais adultos entre 20 e 30 anos (RODRIGUES DC, et al., 2013). 
Apesar da literatura afirmar que a principal queixa do paciente que sofre esse tipo de hipertrofia, é a questão estética, em decorrência da sua assimetria facial, outros sintomas como sensação de peso na região massetérica, dor e moderada limitação da abertura de boca podem estar presentes. A paciente deste caso relatou não se incomodar com a alteração estética, e que a única razão para ter procurado o atendimento foi o quadro sintomatológico, apresentando os sintomas descritos acima (RISPOLI DZ, et al., 2008; RAMOS JER, et al., 2019).

A respeito da etiologia da Hipertrofia, a mesma ainda permanece desconhecida, porém existe uma série de fatores que são considerados contribuintes para o desenvolvimento da mesma, são eles, hábito de mascar chicletes, hábitos parafuncionais (bruxismo, apertamento dentário), distúrbios emocionais, como estresse e ansiedade, microtrauma, distúrbios na ATM, alimentação que necessite de uma maior força mastigatória, mastigação unilateral, má oclusão, retrognatia mandibular e distúrbios no balanço dos neurotransmissores como a dopamina e a acetilcolina (ZORRILLA FCV, et al., 2019; PINTO ASB, et al., 2018).

O caso em questão coincide com tais informações, na qual a paciente revelou possuir o hábito de mastigar mais do lado esquerdo, e que apresentava ansiedade e estresse considerável. A ansiedade e o estresse foram considerados fatores que agravam a intensidade da dor.

Em relação a Artrose, os dados coincidem com achados literários, a paciente se encaixa no perfil mais acometido pela doença, no qual diz que a artrose é mais prevalente no sexo feminino e que a faixa etária mais arremetida tem em média 40 anos de idade. Essa prevalência no sexo feminino sugere uma possível relação a atividade hormonal dos pacientes do sexo feminino (SOARES IS, et al., 2019; FERNÁNDEZ ELL e CULCA FAO, 2019).

Um dos sinais clínicos mais comuns observados nos casos de osteoartrose é a presença de dor, uma limitação no movimento de abertura da boca, crepitação articular durante os movimentos, e também a ausência do aquecimento articular (BANSAL M, 2016). A crepitação, restrição da abertura da boca e a presença de dor, foram observados na paciente em questão, coincidindo com as informações descritas pela literatura.

O diagnóstico da artrose na articulação-temporomandibular é feito através da avaliação clínica, em associação com os exames complementares, como radiografias panorâmicas e tomografias computadorizadas. As radiografias panorâmicas oferecem uma visão lateral do côndilo, dificultando em alguns casos o correto diagnóstico, já as tomografias permitem uma visualização de toda a superfície condilar, permitindo então uma análise mais profunda das estruturas, facilitando o correto diagnóstico da patologia (MAGLIONE HO e LUBERTI RF, 2018). No presente relato de caso, a paciente chegou à clínica já em posse de uma ressonância magnética, na qual possibilitou a correta visualização da patologia e o diagnóstico na mesma sessão.

A artrose tem como principal causa a sobrecarga articular. Alguns dos efeitos causados pela osteoartrose é a destruição da cartilagem da superfície da articulação, remodelação óssea, formação óssea, rarefação e sinovite secundária. Essa patologia representa um processo de adaptação, ocorrendo na mesma a remodelação óssea, essa condição pode se tornar estável, mesmo que a morfologia óssea permaneça alterada, os sintomas normalmente não são relatados pelo paciente (OKESON JP, 2008; ROMERO ME e VELOSO CM, 2017). Em controvérsia a literatura, mesmo após esse processo de remodelação a paciente ainda apresentava sintomatologia dolorosa, sendo necessário então a implementação de um tratamento conservador para a resolução do mesmo.

Essa sintomatologia dolorosa decorrente da Artrose pode ser controlada através da administração de medicamentos anti-inflamatórios não-esteroidais (AINES) ou pelo procedimento de artrocentese (SOARES IS, et al., 2019). E apesar da queixa de dor da paciente, não foi necessário submetê-la ao procedimento de artrocentese ou administração de AINES. A conduta escolhida foi a administração do colágeno tipo II, uma vez ao dia, durante 30 dias, com a finalidade de evitar a destruição das articulações, resolver ou diminuir o quadro sintomatológico e evitar a perda de função da articulação. Os exames complementares a fim da visualização da hipertrofia massetérica revelam um aumento ósseo na região do ângulo mandibular do lado 
afetado pela hipertrofia, provavelmente decorrente da resposta do músculo à situação de hiperatividade (SIMÃO RS, et al., 2014). Coincidindo com tais informações, foi possível notar na radiografia panorâmica do presente caso, as características descritas acima.

Em relação ao tratamento da hipertrofia do músculo masseter, a literatura traz uma variedade de métodos cirúrgicos e não cirúrgicos, os não cirúrgicos incluem aconselhamento psicológico, uso de protetores bucais, relaxantes musculares, drogas ansiolíticas, analgésicos, fisioterapia, restaurações dentárias e ajustes oclusais para corrigir contatos prematuros. Como alternativa cirúrgica, tem a excisão cirúrgica de parte do músculo masseter, com a finalidade de reduzir a assimetria facial, melhorando assim a estética do paciente. Uma alternativa mais conservadora é a aplicação de toxina botulínica do tipo A no masseter, que acarreta a redução do volume muscular (OKESON JP, 2008; ANEHOSUR MDSV, et al., 2020; MANGILI LD, et al., 2006).

Pelo fato de a aplicação da toxina botulínica ser um tratamento em que é necessário a repetição de 6 em 6 meses, por conta de sua reversibilidade, e o método cirúrgico ser um método bem invasivo, e também por conta de a paciente não ter pretensões estéticas, a melhor conduta a ser adotada para esse caso foi a prescrição da fisioterapia do masseter, sendo realizada 10 repetições, três vezes ao dia. A fisioterapia, consiste no paciente fechar suas mãos e com o punho fechado, posicionar as dobras dos dedos (falanges médias) na região massetérica, sempre realizando o movimento de cima para baixo. O ideal é o paciente repita o processo 3 vezes ao dia, e que cada ciclo possua 10 repetições.

\section{REFERÊNCIAS}

1. ANEHOSUR MDSV, et al. Management of Masseter Muscle Hypertrophy and Role of Adjunctive Surgical Procedures. Sage Journals, 2020; 5: 1-7.

2. AYHAN M, et al. Combination of Medical and Surgical Treatments for Masseter Hypertrophy. Hindawi, 2018; 1-5.

3. BANSAL M. Prevalence and diagnostic features of osteoarthrosis of the temporomandibular joint: a review. International Journal of Research in Orthopaedics, 2016; 2(1): 1-4.

4. FERNÁNDEZ ELL, CULCA FAO. Métodos de diagnóstico y tratamiento actuales de la osteoartritis de la articulación temporomandibular: una revisión de la literatura. Rev Cient. Odontol., 2019; 7(1): 121-131

5. GRACIA OPN, et. al. Tratamiento de hipertrofia maseterina unilateral con plano neuromiorelajante y aplicación de toxina botulínica tipo a: caso clínico. Revista OACTIVA UC Cuenca, 2021; 6(1): 59-64.

6. HAGE YE et al. Effect of Facial Massage on Static Balance in Individuals with Temporomandibular Disorder - a Pilot Study. International Journal of Therapeutic massage and bodywork, 2013; 6(4): 6-11.

7. MAGLIONE HO, LUBERTI, RF. Consideraciones clínicas e imagenológicas de la osteoartrosis en la articulación temporomandibular. Revista del Círculo Argentino de Odontologia, 2018; 77(227): 13-17.

8. MANGILI LD, et al. A intervenção fonoaudiológica no pós-operatório da hipertrofia benigna do músculo masseter. Rev. Dent. Press Ortodon. Ortop. Facial, 2006; 11(2): 103-109.

9. NAPHADE VV, et al. Diagnóstico ultrassonográfico de hipertrofia benigna do músculo masseter: relato de caso. J Can Res Ther., 2018; 1237-1240.

10. OKESON JP. Tratamento das desordens temporomandibulares e oclusão, $6^{\circ}$ edição, Rio de Janeiro, Elsevier Editora Ltda, 2008, 348p.

11. PINTO ASB, et al. Evaluation measure of $3 d$ volumetry of masseter hypertrophy: association with modalities of imaging. Rev Gaúch. Odontol., 2018; 66(4): 375-383.

12. RAMOS JER, et al. Tratamento Cirúrgico da Hipertrofia Muscular Massetérica Unilateral: Relato de Caso. Brazilian Journal of Surgery and Clinical Research, 2019; 28(3): 25-28.

13. RISPOLI DZ, et al. Hipertrofia benigna do Músculo masseter. Revista Bras. Otorrinolaringologista, 2008; 74(5): 790793.

14. RODRIGUES, DC et al. Hipertrofia Unilateral do musculo masseter: Relato de Caso. Revista de Cirurgia e T. Bucomaxilo-facial, 2013; 13(4): 21-26.

15. ROMERO ME, VELOSO CM. Diagnóstico de osteoartrosis de articulación temporomandibular con tomografía computada de haz cónico. Revista Digital Founne, 2017; 1:1-8.

16. SÁNCHEZ MT, BECERRA WB. Osteoartritis (artrosis) de la articulación temporomandibular. Rev. Otorrinolaringol. Cir. Cabeza Cuello, 2020; 80: 540-553

17. SIMÃO RS, et al. Hipertrofia benigna do músculo masseter - relato de caso. Revista da Associação paulista de Cirurgiões Dentistas, 2014; 68(4): 351-355.

18. SOARES IS, et al. Artrose na articulação têmporo-mandibular: Relato de caso. Revista ACBO, $2019 ; 8(2)$ : 47-52.

19. VALENCIA AR, et al. Hipertrofia Maseterina Unilateral Idiopática. Revista Espanola de Cirugía Oral y Maxilofacial, 2020; 42(2): 91-93.

20. ZORRILLA FCV, et al. Hipertrofia del Músculo Maserter, Una Revisíon del Escenario Contemporáneo Actual. Rev. Evid. Odontol. Clínic., 2019; 3(2): 50-56. 